\title{
Multiple Feeding Artery Pedicle Pseudoaneurysms in the Posterior Circulation: Association with Hemorrhage and AVM
}

\author{
Soo Mee Lim, MD', Yong Jae Cho, MD², Rena Lee, $\mathrm{PhD}^{3}$
}

We experienced rare combination of multiple irregular shaped aneurysms along the course of the feeding artery and arteriovenous malformation (AVM) in the posterior circulation. We could not explain which aneurysm was a cause of bleeding because all the aneurysms showed irregular in shape like pseudoaneurysms and location of the aneurysms was very close each other. We report two cases in which multiple irregular shaped aneurysms were related with AVMs and first episode of hemorrhage.

Key Words : Aneurysm; Arteriovenous malformation; Embolization

The association between arteriovenous malformations (AVMs) and arterial aneurysms is well known, with an incidence ranging from $2.7 \%$ to $23 \%$ (1). The increase of blood flow in a vessel feeding an AVM predisposes the development of aneurysm on that vessel $(2,3)$, and correlations between the presence of arterial aneurysms and hemorrhage are well established (4). Khayata et al. (5) reported false aneurysms associated with rupture of an AVM and they described false aneurysms probably can be detected more frequently after AVM hemorrhage. The management of the patient with a ruptured AVM should take this morphological feature and location of hematoma into account.

We experienced two cases of rare combination of

Departments of ${ }^{1}$ Radiology, ${ }^{2}$ Neurosurgery and ${ }^{3}$ Radiation Oncology, Ewha Womans University Mokdong Hospital, Seoul, Korea Received April 12, 2010;

accepted after revision May 2, 2010.

Correspondence to: Soo Mee Lim, MD, Department of Radiology, Ewha Womans University Mokdong Hospital, 911-1 Mok-dong Yangcheon-gu, Seoul 158-710, Korea.

Tel. 82-2-2650-5380 Fax. 82-2-2650-5301

E-mail: soomee@ewha.ac.kr

Neurointervention 2011;6:27-30

This is an Open Access article distributed under the terms of the Creative Commons Attribution Non-Commercial License (http://creativecommons.org/licenses/by-nc/3.0) which permits unrestricted non-commercial use, distribution, and reproduction in any medium, provided the original work is properly cited. multiple irregular shaped aneurysms along the course of the feeding artery and AVM in the posterior circulation. We could not explain which aneurysm was a cause of bleeding because all the aneurysms showed irregular in shape like pseudoaneurysms and location of the aneurysms was very close each other. The patients had no history of previous subarachnoid hemorrhage (SAH). To our knowledge, few cases of multiple dysplastic aneurysms and ruptured pseudoaneurysm along the course of arterial feeders with AVMs presented in the literature. We report two cases in which multiple irregular shaped aneurysms were related with AVMs and first episode of hemorrhage.

\section{CASE REPORTS}

\section{Case 1}

First patient was 33-year-old man admitted with generalized tonic-clonic seizure for 5 minutes. The mental status was deep drowsy and there were no neurological deficits. He had no previous history of intracranial hemorrhage. A computed tomography (CT) demonstrated Fisher grade IV SAH along the both sylvian fissures, interhemispheric fissure, and dominantly in the premedullary and prepontine cistern and intraventricular hemorrhage in 3rd and 4th ventricles. 
A digital subtraction angiogram (DSA) revealed two left anterior inferior cerebellar artery (AICA) aneurysms located at the premeatal and meatal branches and two left lateral pontine artery aneurysms and associated AVM distal from the aneurysms (Fig. 1A). The AVM was fed by the dilated left AICA and lateral pontine artery. These aneurysms are all irregular in shape and close to each other. We could not decide which aneurysm was a cause of bleeding.

On following day, these aneurysms and AVM nidus were embolized with $33 \%$ mixture of n-butylcyanoacrylate (NBCA) and Lipiodol (Fig. 1B) at a same time, and residual AVM nidus were treated with stereotactic radiosurgery. 2 years after stereotactic radiosurgery, a small draining vein was remained (Fig.
1C). The patient tolerated well without any neurological deficits.

\section{Case 2}

Second patient was 56-year-old man admitted with stuporous mental status. CT scan demonstrated Fisher grade IV SAH along the both sylvian fissures, basal cistern and intraventricular hemorrhage in 4th ventricle. This hemorrhagic event was first time for the patient.

A DSA revealed three irregular shaped aneurysms located at the meatal, dorsolateral branches of right AICA and AVM fed by the right AICA and right superior cerebellar artery (Fig. 2A). We could not differentiate a ruptured pseudoaneurysm from dysplatic true aneurysms, because the shape of those was similar
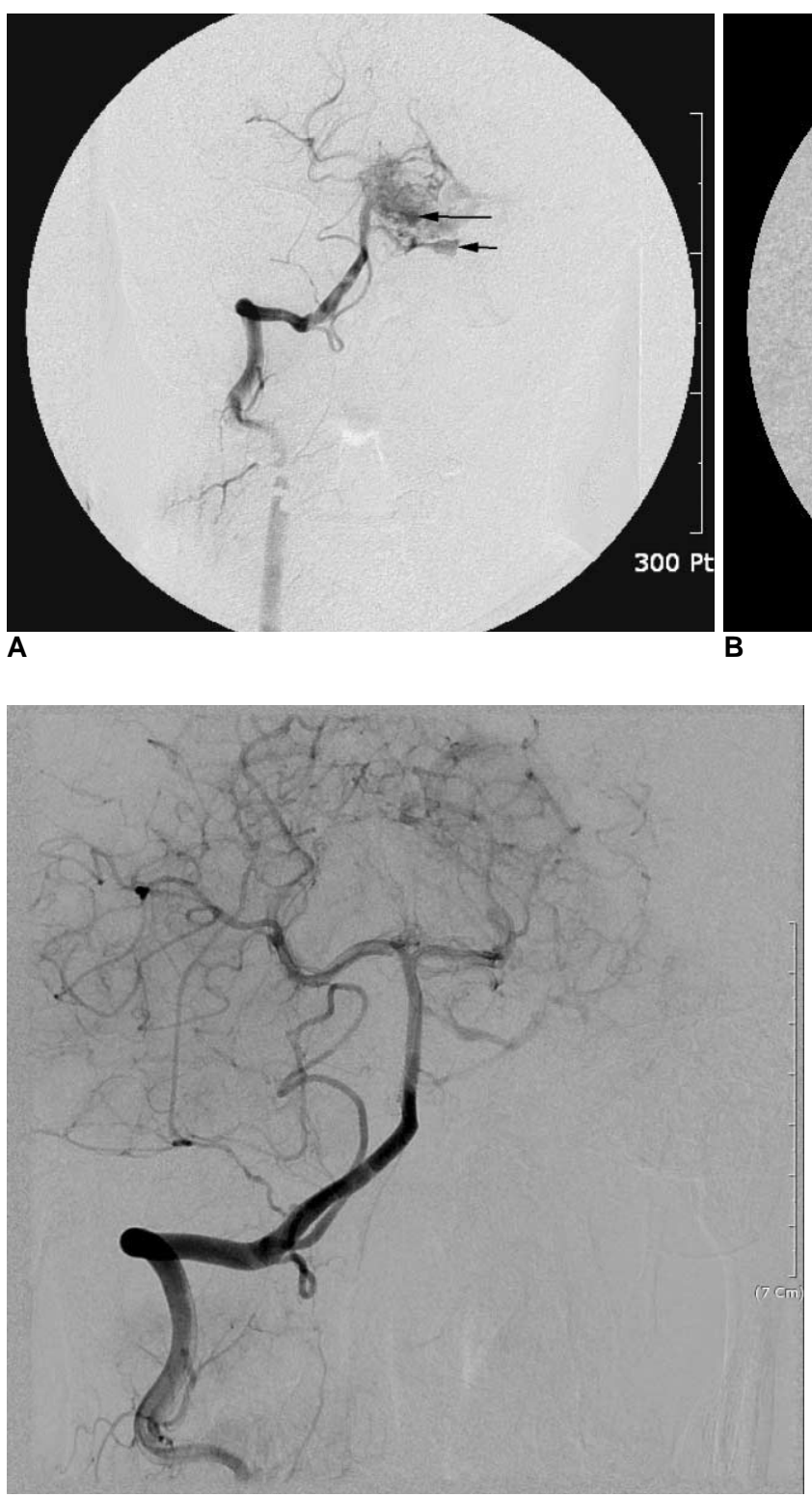

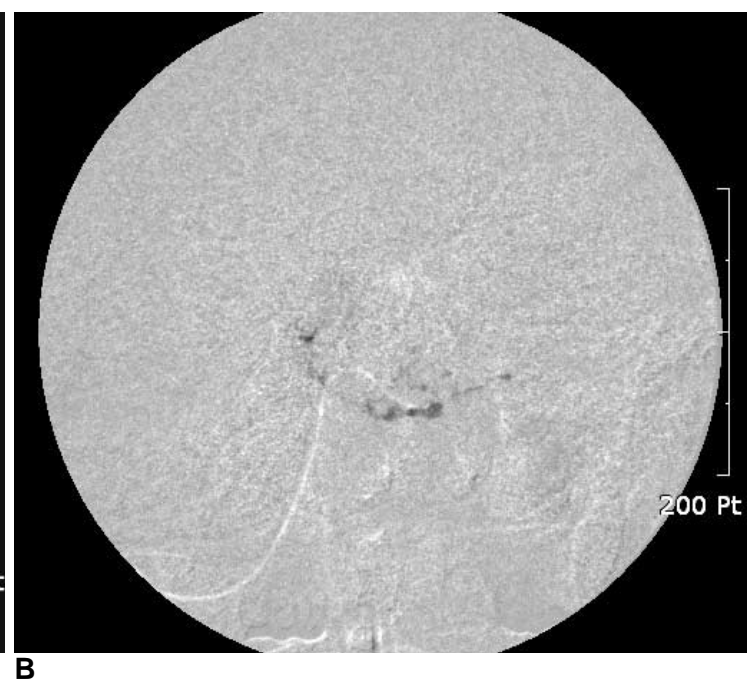

Fig. 1. Case 1

A. Anteroposterior view of right vertebral artery angiogram shows two aneurysms (long arrow) at left pontine artery, two aneurysms (short arrow) in premeatal and meatal segment of left anterior inferior cerebellar artery (AICA) with associated AVM distal from the aneurysms.

B. Two aneurysms and part of AVM feeders of left AICA and left pontine artery are embolized with n-butylcyanoacrylate (NBCA) mixture.

C. Follow up angiogram after NBCA embolization and stereotactic radiosurgery shows a small residual draining vein without definite visible AVM nidus. 

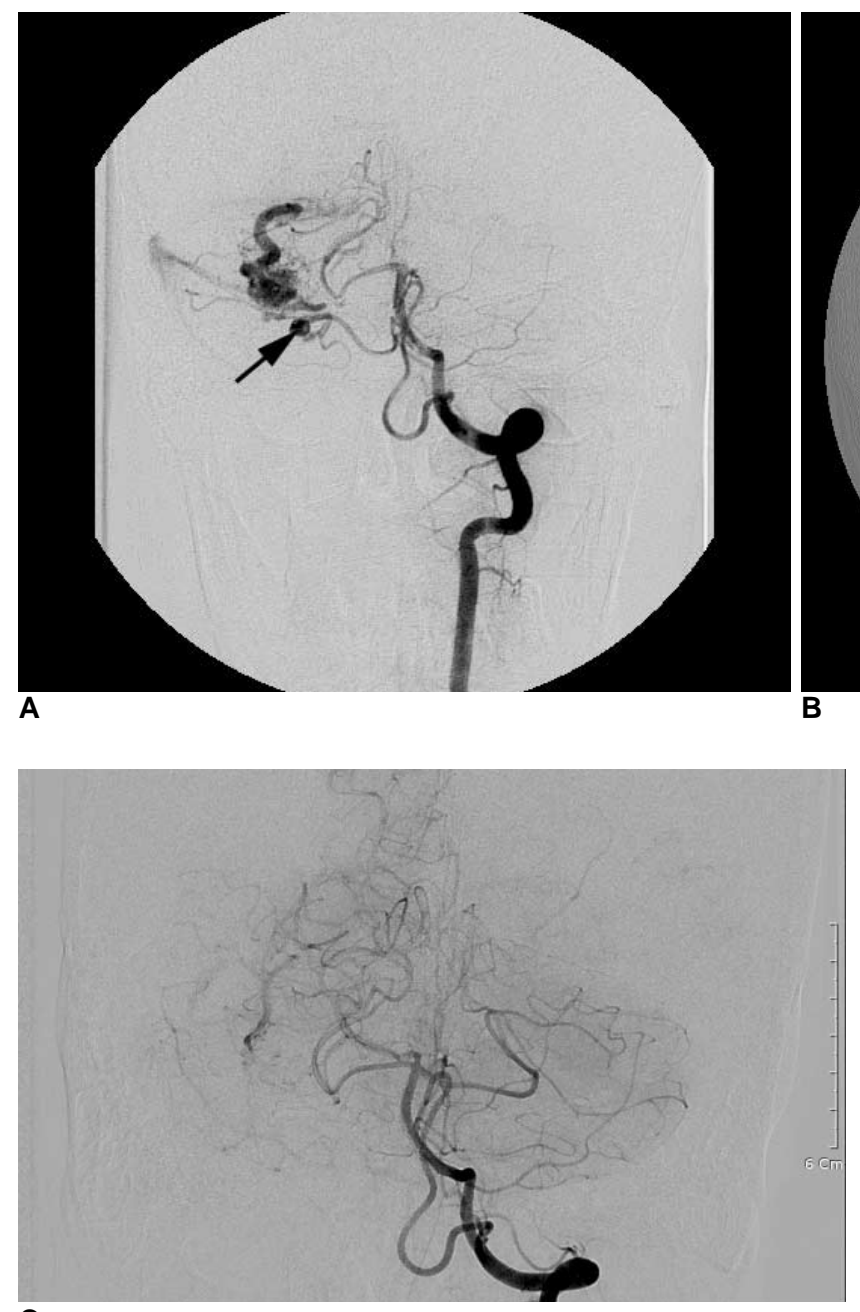

C

and location was very close each other.

On that day, these aneurysms and AVM nidus were embolized (Fig. 2B) with the same procedure as the first patient. Residual AVM nidus was also obliterated with stereotactic radiosurgery. One year after stereotactic radiosurgery, small residual AVM nidus and draining vein were noted (Fig. 2C). There was no episode of recurrent hemorrhage during follow up period.

\section{DISCUSSION}

The occurrence of an intracranial saccular aneurysm of the feeding artery to an AVM is a well-known phenomenon $(2,3)$. The increase of blood flow in a vessel feeding an AVM predisposes the development of aneurysm on that vessel. Marks et al. $(4,6)$ reviewed 65 cases of AVMs that had bled in an attempt to identify which vascular characteristics correlated with past hemorrhage. They identified two types of arterial

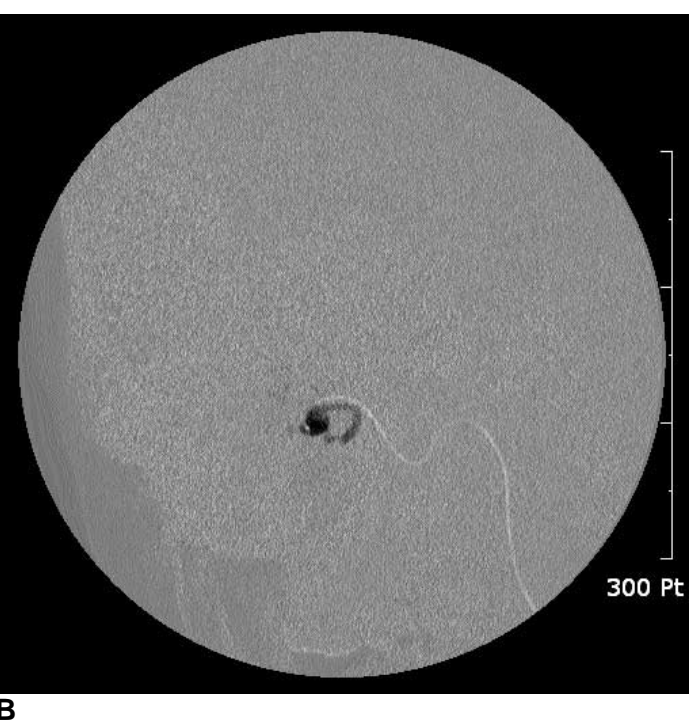

Fig. 2. Case 2

A. Anteroposterior view of left vertebral artery angiogram shows two aneurysms (arrow) at the feeding pedicle of anterior inferior cerebellar artery (AICA) and peripheral AVM fed by the AICA and superior cerebellar artery.

B. Road map image shows NBCA cast at aneurysms of right AICA and feeder of AVM.

C. 1 year follow up angiogram after NBCA embolization and stereotactic radiosurgery shows residual small AVM nidus and draining vein in right cerebellar region. aneurysms: 1) intranidal aneurysms that show a high association with hemorrhage and 2) arterial aneurysms in either the circle of Willis or an arterial pedicle supplying the AVM. However, the latter was not distinguished as an important source of hemorrhage. Perata et al. (7) attempted to clarify these two classifications, and defined pedicle aneurysms as those along the course of the feeding artery but remote from the circle of Willis. They classified the aneurysms as 1) dysplastic or remote, unrelated to inflow vessels, 2) proximal, arising at the circle of Willis origin of a vessel supplying the AVM, 3) pedicular, arising from the midcourse of a feeding pedicle, and 4) intranidal, within the AVM nidus itself. They had question whether these pedicle aneurysms are true aneurysms that were present prior to bleeding or pseudoaneurysms developing when a rupture of a weak, thin-walled vessel occurs. In their cases, four patients suffered recurrent hemorrhage before surgery and aneurysm causing hemorrhage was a single one in each cases. But in our cases, the 
aneurysms on the feeding artery pedicles adjacent to the AVM were multiple and patients had no history of previous hemorrhagic event. Therefore, it was not easy to point out ruptured pseudoaneurysm from residual ones. The differential diagnosis among aneurysms, a true nidal aneurysm, and a pseudoaneurysm is not easy to make except by pathological evidence $(8,9)$.

Despite imperfect knowledge of the exact etiology and histology of pedicle aneurysms, these aneurysms are an important subgroup of aneurysms associated with AVMs and indicate a site of hemorrhage $(4,6)$.

It should become the focus of attention in relation to treatment. Discovery of a pedicle aneurysm should prompt aggressive management of the aneurysm itself with embolization when AVM resection must be delayed or when radiosurgery is planned.

In our cases, the aneurysms were found in the near the meatal segment of the AICA ( 1 in premeatal, 3 in meatal, and 1 in dorsolateral segment) and proximal pontine artery. Andaluz et al. (10) reported that 76.2\% of aneurysms of the AICA were located in the meatal segment and only 10 of 86 were associated with AVMs. Among three patients who had multiple peripheral AICA aneurysms, two were associated with cerebellar AVMs, and one patient had two aneurysms in the distal dorsolateral branch of the AICA. In our cases, $60 \%$ of the aneurysms were in meatal segment of AICA and we guess these aneurysm formation can be related with turbulent flow by acute angulation of this segment.

We suspect one of aneurysms must be pseudoaneurysm due to its association with recent hemorrhage. However, it was not easy to differentiate ruptured pseudoaneurysm from dysplastic flow related true aneurysms.

We attempted endovascular embolization with NBCA to obliterate all the aneurysms and to restrict AVM blood flow at a same time. After control of feeding pedicle having aneurysms, embolization of the other pedicles of AVM was done. Control of the aneurysms prior to the embolization of AVM feeders may avoid the catastrophic consequences of re- bleeding. Residual AVM was amenable to radiosurgery. In conclusion, we experienced two cases of multiple feeding pedicle aneurysms associated with posterior fossa AVMs presented with a one episode of SAH. These patients had no history of previous intracranial hemorrhage but all aneurysms had irregular shape like pseudoaneurysms. The differentiation of ruptured aneurysm from unruptured aneurysms was not possible. Embolization of aneurysms and AVM nidus with NBCA at a same time showed good clinical results. In cases of having remained AVM nidus, radiosurgery is recommended to obliterate the residual AVM nidus.

\section{References}

1. Lasjaunias P, Piske R, Terbrugge K, Willinsky R. Cerebral arteriovenous malformations (C. AVM) and associated arterial aneurysms (AA). Analysis of $101 \mathrm{C}$. AVM cases, with 37 AA in 23 patients. Acta Neurochir (Wien) 1988;91:29-36

2. Cockroft KM, Thompson RC, Steinberg GK. Aneurysms and arteriovenous malformations. Neurosurg Clin N Am 1998;9:565576

3. Thompson RC, Steinberg GK, Levy RP, Marks MP. The management of patients with arteriovenous malformations and associated intracranial aneurysm. Neurosurgery 1998;43:202-211

4. Marks MP, Lane B, Steinberg GK, Chang PJ. Hemorrhage in intracerebral arteriovenous malformations: angiographic determinants. Radiology 1990;176:807-813

5. Khayata MH, Zabramski JM, Johnson PC, Flom R. False aneurysm associated with rupture of an arteriovenous malformation-implication for treatment: case report. Neurosurgery 1993;33:753-756

6. Marks MP, Lane B, Steinberg GK, Snipes GJ. Intranidal aneurysms in cerebral arteriovenous malformations: evaluation and endovascular treatment. Radiology 1992;183:355-360

7. Perata HJ, Tomsick TA, Tew JM Jr. Feeding artery pedicle aneurysms: association with parenchymal hemorrhage and arteriovenous malformation in the brain. J Neurosurg 1994;80:631-634

8. Blackford JM, Mclaughlim JS. Pseudoaneurysms of the carotid artery. Am Surg 1973;39:257-260

9. Nayeem SA, Tada Y, Takagi A, Sato O, Miyata T, Idezuki Y. Carotid artery pseudoaneurysm following internal jugular vein cannulation. J Cardiovasc Surg (Torino) 1990;31:182-183

10. Andaluz N, Pensak ML, Zuccarello M. Multiple, peripheral aneurysms of the anterior inferior cerebellar artery. Acta Neurochir (Wien) 2005;147:419-422 